\title{
ClearDetect: disposable colorimetric sensors for rapid and presumptive identification of CWA and advanced threats (Withdrawal Notice)
}

\section{Madeline Vara, Piercen Oliver}

Madeline Vara, Piercen Oliver, "ClearDetect: disposable colorimetric sensors for rapid and presumptive identification of CWA and advanced threats

(Withdrawal Notice)," Proc. SPIE 11749, Chemical, Biological, Radiological, Nuclear, and Explosives (CBRNE) Sensing XXII, 1174906 (12 April 2021); doi: $10.1117 / 12.2588357$

SPIE. Event: SPIE Defense + Commercial Sensing, 2021, Online Only 


\section{ClearDetect disposable colonimetric sensors for rapid and presumptive identification of CWA and advanced threats (Withdrawal Notice)}

Madeline Vara, and Piercen Oliver

Clear Scientific, Inc. (United States)

Proceedings Volume 11749, Chemic al, Biologic al, Radiological, Nuc lear, and Explosives (CBRNE) Sensing XXIl; 1174906 (2021) https:// doi.org/10.1117/12.2588357

Event SPIE Defense + Commercial Sensing Digital Forum, 2021, O nline Only, United States

Online Publication Date: 13 April 2021

Withdrawn from Publication: 4 May 2021

Publisher's Note: This pa per, orig ina lly published on 13 April 2021, was withdrawn per author request. 